\title{
APPROXIMATING THE ABSOLUTELY CONTINUOUS MEASURES INVARIANT UNDER GENERAL MAPS OF THE INTERVAL
}

\author{
ABRAHAM BOYARSKY ${ }^{1}$
}

\begin{abstract}
Let $\tau: I \rightarrow I$ be a nonsingular, piecewise continuous transformation which admits a unique absolutely continuous invariant measure $\mu$ with density function $f^{*}$. The main result establishes the fact that $f^{*}$ can be approximated weakly by the density functions of a sequence of measures invariant under piecewise linear Markov maps $\left\{\tau_{n}\right\}$ which approach $\tau$ uniformly.
\end{abstract}

1. Introduction. Let $\tau$ be a nonsingular, measurable transformation from $I=[0,1]$ into itself and let $\mathbb{B}_{\mathrm{B}}$ denote the Lebesgue measurable subsets of $I$. A measure $\mu$ defined on $(I, 9)$ is absolutely continuous if there exists a function $f: I \rightarrow[0, \infty)$, which is integrable with respect to Lebesgue measure $m$, i.e., $f \in L_{1}^{\prime}\left(I, \frac{1}{h}, m\right) \equiv L_{1}^{\prime}$, and for which

$$
\mu(S)=\int_{S} f(x) m(d x) \quad \forall S \in \text { 约. }
$$

The measure $\mu$ is said to be invariant (under $\tau$ ) if $\mu\left(\tau^{-1} S\right)=\mu(S)$ for all sets $S \in$ of .

The Frobenius-Perron operator $P_{\tau}: P_{1} \rightarrow L_{1}$ has proven to be a useful tool in the study of absolutely continuous invariant measures [1,2]. It is defined by

$$
\left(P_{\tau} f\right)(x)=\frac{d}{d x} \int_{\tau^{-1}[0, x]} f(s) m(d s) .
$$

The importance of $P_{\tau}$ lies in the fact that each of its fixed points is the density of a measure invariant under $\tau$, i.e., if $P_{\tau} f^{*}=f^{*}$, then

$$
\mu(\quad)=\int f^{*}(x) m(d x)
$$

is invariant under $\tau[\mathbf{1}]$.

In [2] a sequence of matrices $\left\{P_{n}\right\}$, depending on $\tau$, is constructed and the following result obtained:

THEOREM 1. Let $\tau: I \rightarrow I$ be a piecewise $C^{2}$ map with inf $\left|\cdot \tau^{\prime}\right|>2$. If $P_{\tau}$ has a unique fixed point $f^{*}$, then the sequence $\left\{f_{n}\right\}$ of fixed points (regarded as functions on $I$ ) of $\left\{P_{n}\right\}$ converges to $f^{*}$ in the $\mathcal{E}_{1}$-norm.

Received by the editors March 30, 1982.

1980 Mathematics Subject Classification. Primary 28D05; Secondary 41A30.

'This research was supported by NSERC Grant No. A-9072 and an FCAC Grant from the Education Department of Quebec. 
The proof of Theorem 1 depends on the fact that $P_{\tau}$, where $\tau$ is expanding, reduces the variation of the function on which it acts [1]. The critical inequality is

$$
\mathrm{V}_{0}^{\mathrm{V}} P_{\tau} f \leqslant \alpha\|f\|+\beta \mathbf{V}_{0}^{\mathrm{I}} f,
$$

where $\mathrm{V}_{0}^{\prime}$ denotes the variation on $[0,1]$ and \|\| the $E_{1}$-norm. In the proof of Theorem $1, \beta$ must be less than 1 and this is truly only when inf $\left|\tau^{\prime}\right|>2$.

When $\tau$ is nonexpanding, as for example if $\tau(x)=\gamma x(1-x)$, where $\gamma$ can take on any value between 0 and 4 , there are no known results similar to Theorem 1 . The technique of [2] fails because $P_{\tau} f$ can have infinite variation for $f$ of bounded variation.

In this note we shall obtain a result analogous to Theorem 1 for a large class of transformations $\tau$ which admit a unique absolutely continuous invariant measure. To do this we shall use the weak topology on the space of measures and the result will be of the form: $f_{n} \stackrel{\omega}{\rightarrow} f^{*}$, where $\omega$ denotes weak convergence. Although this may not appear to be a strong result, it is sufficient for most statistical purposes; for example, all the moments of the density $f_{n}$ will be close to the corresponding ones for $f^{*}$, and

$$
\mu_{n}(S)=\int_{S} f_{n}(x) m(d x) \rightarrow \int_{S} f^{*}(x) m(d x) \equiv \mu(S)
$$

for any $S \in \cup B$.

2. Notation. A piecewise continuous map $\tau_{n}: I \rightarrow I$ is called Markov if there exist points $a_{0}<a_{1}<\cdots<a_{n-1}<a_{n}$ such that for $i=0,1, \ldots, n-1,\left.\tau\right|_{l}$, where $I_{i}=$ $\left(a_{i-1}, a_{i}\right)$, is a homeomorphism onto some interval $\left(a_{j(i)}, a_{k(i)}\right)$. The partition $J_{n}=\left\{I_{1}^{n}\right\}_{1=1}^{n}$ is referred to as a Markov partition with respect to $\tau$.

Now let $\tau: I \rightarrow I$ be piecewise continuous and nonsingular. Partition $I \times I$ into an $n \times n$ grid and form the piecewise linear map $\tau_{n}$ by joining corner points of the grid in such a way that $\tau_{n}$ approximates $\tau$. Clearly $\tau_{n}$ will have only integer slopes and $\tau_{n} \rightarrow \tau$ as $n \rightarrow \infty$ in the uniform norm.

The Frobenius-Perron operator $P_{\tau_{n}}$, when restricted to step functions on $J_{n}$, can be represented by a matrix [6], which we denote by $P_{n}$; its entries are given by

$$
\begin{aligned}
p_{i j}^{n} & =1 /\left.\tau_{n}^{\prime}\right|_{I_{i}} \quad \text { if } I_{j}^{n} \subset \tau_{n}\left(I_{i}^{n}\right), \\
& =0 \text { otherwise. }
\end{aligned}
$$

In [3] it is shown that $P_{n}$ is similar to a stochastic matrix and therefore has a fixed point $f_{n}$, which we regard as a step function on $J_{n}$. Our aim is to prove that $f_{n}$ converges weakly to $f^{*}$, the density of the unique measure invariant under $\tau$.

DEFINITION 1. Let $\left\{\mu_{n}\right\}$ be a sequence of absolutely continuous probability measure on $(I, \mathscr{B})$ and let $f_{n}$ be the density of $\mu_{n}$. We shall say that $f_{n}\left(\mu_{n}\right)$ converges weakly to the density $f$ (measure $\mu$ ) if and only if for each $g \in C$, the space of real, bounded and continuous functions on $I$,

$$
\int_{I} g(x) f_{n}(x) m(d x) \rightarrow \int_{I} g(x) f(x) m(d x)
$$

as $n \rightarrow \infty$. 
In fact it is sufficient that $g$ is in a space $D$ dense in $C$ [4, Theorem 12.2]. For our purposes, we shall use $C^{1}$, the space of functions on $I$ which have continuous first derivatives.

Definition 2. Let $g$ be any function from $I$ into $(-\infty, \infty)$, and let $\delta$ and $\varepsilon$ be positive numbers. We denote by $\partial_{\delta, e}(g)$ the set of those points $x \in I$ for which the distance between $g\left(x^{\prime}\right)$ and $g\left(x^{\prime \prime}\right)$ exceeds $\varepsilon$ for some pair of points $x^{\prime}, x^{\prime \prime}$ in the open interval $(x-\delta, x+\delta)$.

A more general version of the following theorem is proved in [5].

THEOREM 2. Let $\left\{g_{n}\right\}_{n>1}$ be a sequence of bounded, real-valued and measurable functions defined on $S$ and let $\alpha$ be a real number. Then a necessary and sufficient condition that $\int_{I} g_{n}(x) f_{n}(x) m(d x) \rightarrow \alpha$ for every sequence $\left\{f_{n}\right\}$ converging weakly to $f$ is that

(i) $\left\{g_{n}\right\}_{n \geq 1}$ is uniformly bounded,

(ii) $\int_{l} g_{n}(x) f(x) m(d x) \rightarrow \alpha$ and

(iii) $\forall \varepsilon>0, \lim _{\delta \rightarrow 0} \lim \sup _{n \rightarrow \infty} \int_{\partial_{\delta, e}\left(g_{n}\right)} f(x) m(d x)=0$.

It can be shown that (iii) holds iff

(iii') $\forall \varepsilon>0$, for every sequence $\left\{\delta_{k}\right\}$ of positive numbers converging to 0 , and for every subsequence $\left\{g_{n_{k}}\right\}, \int_{n_{k=1}^{\infty} \partial_{\delta_{k}, r}\left(g_{n_{k}}\right)} f(x) m(d x)=0$.

LEMMA 1 . Let $g$ be a bounded, piecewise continuous function on $[0,1]$ whose set of discontinuity points, $D$, has Lebesgue measure 0 . Let $\left\{g_{n}\right\}$ be a uniformly bounded sequence of piecewise continuous functions which approaches $g$ uniformly. Then, if $f_{n} \stackrel{\omega}{\rightarrow} f$,

$$
\int_{I} g_{n}(x) f_{n}(x) m(d x) \rightarrow \int_{I} g(x) f(x) m(d x)
$$

as $n \rightarrow \infty$.

Proof. Since $g_{n} \rightarrow g$ uniformly, we have

$$
\int_{I} g_{n}(x) f(x) m(d x) \rightarrow \int_{I} g(x) f(x) m(d x)=\alpha .
$$

It remains to prove (iii'). Let $\varepsilon>0$. Then for any sequence $\left\{\delta_{k}\right\}$ of positive numbers converging to 0 and every subsequence $\left\{g_{n_{k}}\right\}, \cap_{k=1}^{\infty} \partial_{\delta_{k}, \varepsilon}\left(g_{n_{k}}\right) \subset D$. Since $m(D)=0$, (iii') is valid and Theorem 2 can be invoked. Q.E.D.

\section{Main result.}

LEMMA 2. Let $\left\{\tau_{n}\right\}$ be a sequence of nonsingular transformations from $I \rightarrow I$ which approach $\tau$ uniformly. Let $f \in \varrho_{1}$. Then

$$
\int_{I} h(x)\left(P_{\tau_{n}} f\right)(x) m(d x) \rightarrow \int_{I} h(x)\left(P_{\tau} f\right)(x) m(d x)
$$

as $n \rightarrow \infty$ for any $h \in C^{\prime}$. 
Proof. From the definition of the Frobenius-Perron operator, we have

$$
\begin{aligned}
\int_{I} h(x)\left(P_{\tau_{n}} f(x)-P_{\tau} f(x)\right) m(d x) \\
\quad=\int_{I} h(x)\left\{\frac{d}{d x} \int_{\tau_{n}^{-1}[0, x]} f(y) m(d y)-\frac{d}{d x} \int_{\tau^{-1}[0, x]} f(y) m(d y)\right\} m(d x) .
\end{aligned}
$$

Integrating by parts,

$$
\begin{aligned}
\int_{0}^{1} h(x)\left(\frac{d}{d x} \int_{\tau^{-1}[0, x]}\right. & f(y) m(d y)) m(d x) \\
= & g(1) \int_{0}^{1} f(y) m(d y)-\int_{0}^{1} \int_{\tau^{-1}[0, x]} f(y) m(d y) g^{\prime}(x) m(d x) .
\end{aligned}
$$

Thus,

$$
\begin{aligned}
\int_{I} h(x)\left(P_{\tau_{n}} f(x)\right. & \left.-P_{\tau} f(x)\right) m(d x) \\
& =\int_{I}\left\{\int_{\tau^{-1}[0, x]} f(y) m(d y)-\int_{\tau_{n}^{-1}[0, x]} f(y) m(d y)\right\} h^{\prime}(x) m(d x)
\end{aligned}
$$

and

$$
\begin{aligned}
& \mid \int_{l} h(x)\left(P_{\tau_{n}} f(x)\right.\left.-P_{\tau} f(x)\right) m(d x) \mid \\
& \leqslant \int_{l} \int_{\left(\tau^{-1}[0, x]\right) \Delta\left(\tau_{n}^{-1}[0, x]\right)}|f(y)| m(d y) h^{\prime}(x) m(d x),
\end{aligned}
$$

where $\triangle$ denotes the symmetric difference. Since $\tau_{n} \rightarrow \tau$ uniformly as $n \rightarrow \infty$, $m\left\{\left(\tau^{-1}[0, x]\right) \triangle\left(\tau_{n}^{-1}[0, x]\right)\right\} \rightarrow 0$ as $n \rightarrow \infty$. Since $h^{\prime}(x)$ is continuous on $I$, it is bounded. This completes the proof. Q.E.D.

We can now state the main result of this note.

THEOREM 3. Let $\tau: I \rightarrow I$ be a nonsingular, piecewise continuous map, whose set of discontinuities has Lebesgue measure 0 , and let $\tau$ admit a unique absolutely continuous probability measure $\mu$. Let $\left\{\tau_{n}\right\}$ be a sequence of piecewise linear Markov maps which approach $\tau$ uniformly. Let $f_{n}$ denote a fixed point of $P_{n} \equiv P_{\tau_{n}}$, where $\left\|f_{n}\right\|=1$ and $f_{n}>0$. Then $f_{n} \stackrel{\omega}{\rightarrow} f^{*}$ as $n \rightarrow \infty$, where $f^{*}$ is the density function of $\mu$.

Proof. Since $I$ is compact, the family of probability measures $\left\{\mu_{n}\right\}$, defined by $\mu_{n}(E)=\int_{E} f_{n}(x) m(d x)$, is weakly compact. Hence there exists a subsequence $\left\{f_{n_{1}}\right\}$ and a function $f: I \rightarrow I$ such that $f_{n} \stackrel{\omega}{\rightarrow} f$.

Now, for any $h \in C^{\prime}$,

$$
\begin{aligned}
& \left|\int_{I} h(x)\left(f(x)-P_{\tau} f(x)\right) m(d x)\right| \\
& \quad \leqslant\left|\int_{I} h(x)\left(f(x)-f_{n_{1}}(x)\right) m(d x)\right|+\left|\int_{I} h(x)\left(f_{n_{1}}(x)-P_{n_{1}} f_{n_{1}}(x)\right) m(d x)\right| \\
& \quad+\left|\int_{I} h(x)\left(P_{n_{1}} f_{n_{1}}(x)-P_{n_{1}} f(x)\right) m(d x)\right|+\left|\int_{I} h(x)\left(P_{n_{1}} f(x)-P_{\tau} f(x)\right) m(d x)\right| .
\end{aligned}
$$


The first term approaches 0 since $f_{n_{1}} \stackrel{\omega}{\rightarrow} f$. Since $P_{n_{1}} f_{n_{1}}=f_{n_{i}}$, the second term is identically 0 . The fourth term approaches 0 by virtue of Lemma 2. Consider now the third term,

$$
\begin{aligned}
\int_{0}^{1} h(x) \frac{d}{d x}\left\{\int _ { \tau _ { n _ { 1 } } ^ { - 1 } [ 0 , x ] } \left(f_{n_{1}}(y)\right.\right. & -f(y)) m(d y)\} m(d x) \\
= & \int_{0}^{1}\left\{\int_{\tau_{n}^{-1}[0, x]}\left[f_{n_{1}}(y)-f(y)\right] m(d y)\right\} h^{\prime}(x) m(d x) .
\end{aligned}
$$

Fix $x \in[0,1]$ and consider

$$
\begin{aligned}
& A_{n_{t}}(x) \equiv \int_{\tau_{n_{t}}^{-1}[0 . x]}\left[f_{n_{t}}(y)-f(y)\right] m(d y) \\
& =\int_{I} \chi_{\tau_{n_{i}}^{-1}[0, x]}(y) f_{n_{i}}(y) m(d y)-\int_{I} \chi_{\tau_{n_{i}}^{-1}\{(0, x]}(y) f(y) m(d y) .
\end{aligned}
$$

Now $\chi_{\tau_{n}^{-1}(0, x)}(y)$ is a piecewise continuous step function which approaches $\chi_{\tau^{-1}[0, x]}$ uniformly as $n_{i} \rightarrow \infty$. Clearly

$$
\int_{I} \chi_{\tau_{n}^{-1},[0, x]}(y) f(y) m(d y) \rightarrow \int_{I} \chi_{\tau^{-1}[0, x]}(y) f(y) m(d y) \equiv \alpha
$$

as $n_{i} \rightarrow \infty$. Thus, it follows from Lemma 1 that $A_{n_{i}}(x) \rightarrow 0$ as $n_{i} \rightarrow \infty$. Note that $\left|A_{n_{1}}(x)\right| \leqslant 2$. Since $h \in C^{\prime},\left|h^{\prime}(x)\right| \leqslant L<\infty$. Hence, the Dominated Convergence Theorem implies that

$$
\int_{0}^{1} A_{n_{1}}(x) h^{\prime}(x) m(d x) \rightarrow 0
$$

as $n_{i} \rightarrow \infty$. We have, therefore, established, for any $h \in C^{\prime}$,

$$
\int_{I} h(x)\left(f(x)-P_{\tau}(x)\right) m(d x)=0 .
$$

This means $P_{\tau} f(x)=f(x) m$-a.e. But $f^{*}$ is the unique fixed point of $P_{\tau}$. Thus $f=f^{*}$ $m$-a.e., and $f_{n} \stackrel{\omega}{\rightarrow} f^{*}$. We have therefore shown that any weakly convergent subsequence of $\left\{f_{n}\right\}$ converges weakly to $f$. Hence $f_{n} \stackrel{\omega}{\rightarrow} f$ as $n \rightarrow \infty$. Q.E.D.

REMARKS. (1) Theorem 1 establishes a necessary condition for the existence of an absolutely continuous invariant for a general map $\tau: I \rightarrow I$.

(2) Classes of maps $\tau: I \rightarrow I$ which are nonexpanding. and which have unique absolutely continuous invariant measures can be found in [7-10]. Theorem II.8.3 of [10] describes some of the results in [7].

\section{REFERENCES}

1. A. Lasota and J. A. Yorke, On the existence of invariant measures for piecewise monotonic transformations, Trans. Amer. Math. Soc. 186 (1973), 481-488.

2. T.-Y. Li, Finite approximation for the Frobenius-Perron operator. A solution to Ulam's conjecture, J. Approx. Theory 17 (1976), 177-186.

3. N. Friedman and A. Boyarsky, Matrices and eigenfunctions induced by Markov maps, Linear Algebra Appl. 38 (1981), 141-147.

4. G. Choquet, Lectures on analysis, Vol. I, Benjamin, New York, 1969. 
5. F. Topsoe, Preservation of weak convergence under mappings, Ann. Math. Statist. 38 (1967), 1661-1665

6. A. Boyarsky and M. Scarowsky, On a class of transformations which have unique absolutely continuous invariant measures. Trans. Amer. Math. Soc. 255 (1979), 243-262.

7. M. Misiurewicz, Absolutely continuous invariant measures for certain maps of the interval. Inst. Hautes Études Sci. Publ. Math. 52 (1980).

8. D. Ruelle, Applications conservant une mesure absolument continue par rapport à dx sur [0, 1]. Comm. Math. Phys. 55 (1977), 47-51.

9. R. Bowen, Invariant measures for Markov maps of the interval, Comm. Math. Phys. 69 (1979), 1-17.

10. P. Collet and J.-P. Eckmann. Iterated maps of the interval, Birkhauser, Boston, Mass., 1980.

Department of Mathematics, loyola Campus, Concordia University, Montreal, Canada H4B IR6 